\title{
A Study Of Morphometrical Differences Between Normal Mucosa, Dysplasia , Squamous Cell Carcinoma \& Pseudoepitheliomatous Hyperplasia Of The Oral Mucosa
}

\author{
Upadhyaya $\mathrm{Neha}^{1}$, Khandekar Shubhangi $^{2}$, Dive Alka ${ }^{3}$,Mishra Rakesh \\ Kumar $^{4}$,Moharil Rohit ${ }^{5}$ \\ ${ }^{1}$ (Department of Oral \& Maxillofacial Pathology, V.S.P.M.D.C.R.C,Nagpur,India) \\ ${ }^{2}$ (Department of Oral \& Maxillofacial Pathology, V.S.P.M.D.C.R.C, Nagpur,India) \\ ${ }_{3}^{3}$ (Department of Oral \& Maxillofacial Pathology, V.S.P.M.D.C.R.C, Nagpur,India) \\ ${ }^{4}$ (Department of Ear,Nose \& Throat,N.K.P.S.I.M.S,Nagpur,India) \\ ${ }_{5}^{5}$ (Department of Oral \& Maxillofacial Pathology, V.S.P.M.D.C.R.C,Nagpur,India)
}

\begin{abstract}
Cancer is a highly complex disease due to the disruption of tissue architecture. Thus, tissues, and not individual cells, are the proper level of observation for the study of carcinogenesis. Thus the shape and regularity of the epithelial connective tissue interface(ECTI), has classically been used to describe both physiological and pathological changes in the oral mucosa,including cancer. However, the description of ECTI has most often been subjective. The use of fractal geometry, has therefore been used to describe the irregularities of ECTI profiles taken from the oral mucosa both in normal and abnormal cases.Also the Pseudoepitheliomatous hyperplasia (PEH) of the covering epithelium mimics the squamous cell carcinoma invasion.PEH cells do not show marked atypia , but the presence of pseudoinvasive pattern remains a diagnostic problem since several misdiagnosed cases have been reported. Similar diagnostic problems do occur sometimes between normal \& dysplasia or dysplasia \& squamous cell carcinoma. The aim of this study is to investigate the objective morphometric criteria to distinguish each entity separately ie. normal ,dysplasia ,pseudoepitheliomatous hyperplasia \& squamous cell carcinoma .
\end{abstract}

Keywords: oral mucosa, squamous cell carcinoma, fractal analysis, complexity, tumor shape

\section{Introduction:}

The shape of an object can be used to measure its complexity and like any other hierarchical construct, biological entities are thought to potentially adopt an undefined possibility of configurations ("forms") inside the realm of a common general frame. However, only a limited number of them is actually observed.This can be exemplified by the protein structures, where the number of folds is the transfinite number of possible dispositions of $\mathrm{N}$ residues in space; different sequences may give rise to the same fold, resulting in the configuration that can be considered as the integrated output of such complex and dynamic interplay taking place at the microscopic level. Therefore, shape descriptors can be reliably used as overall indicators of the macro states. Indeed, cancer diagnosis as well as that of other diseases has been routinely made by looking at the cell and tissue shapes, elicited by these pathological entities ${ }^{1}$.The shape and regularity of the epithelial connective tissue interface (ECTI) . "Fig. 1" has classically been used to describe both physiological and pathological changes in the oral mucosa. However, this description is subjective. The irregularities of ECTI profiles can be estimated quantitatively by the use of fractal geometry principles ${ }^{2}$.

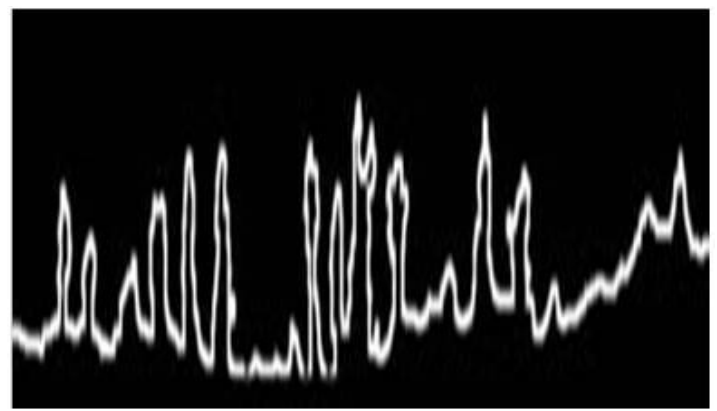

Figure 1 


\section{What Is Fractal Geometry? :}

Classical Euclidean Geometry describes properties of regular smooth shaped objects such as circles or squares by using their perimeter. These Euclidean descriptions are inadequate for complex irregular-shaped objects that occur in nature (e.g. clouds, coastlines and biological structures). These "non- Euclidean" objects are better described by fractal geometry which has the ability to quantify the irregularity and complexity of objects with a measurable value called the fractal dimension. Fractal dimension differs from our intuitive notion of dimension in that it can be a non-integeral value.The more irregular and complex an object is the higher is its fractal dimension. An image is called "fractal" if it displays self-similarity and it can be split into parts, each of which is (at least approximately) a reduced-size copy of the whole. A possible calculation of a fractal set is provided by the "box-counting" method. The number $\mathbf{N}$ of boxes of size $\mathbf{R}$ needed to cover a fractal set follows a power-law, $\mathrm{N}=\mathrm{N} 0 * \mathrm{R}^{\wedge}(-\mathrm{DF})$, with $\mathrm{DF}<=\mathrm{D}$ ( $\mathrm{D}$ is the dimension of the space, usually $\mathrm{D}=1,2,3$ ), which is repeated with different box sizes.

\section{"Fractal dimension $=$ slope $=(\mathrm{d} \ln \mathrm{N} / \mathrm{d} \ln \mathrm{R}) / \mathrm{R} "$}

\section{Aim And Objective :}

The aim of this study is to use fractal geometry to compare the morphometric complexity in normal,dysplastic, malignant \& pseudo-epitheliomatous hyperplasia of the oral mucosa. It will be fulfilled by estimating the fractal dimensions of ECTI profiles isolated from histological sections representing these different entities.

Hence proposing fractal dimension a quantitative marker of epithelial complexity,which can be used as a useful morphological marker of tissue complexity changes taking place during epithelial malignancy and premalignancy. ${ }^{3,4,5}$

\section{Material \& Method :}

4.1, H\&E Stained histological images at $4 \mathrm{X}$ (Scanner View) for:
4.1.1, Normal Epithelium
(Figure 2)
(Figure 3)
10 slides
4.1.2, Dysplastic Epithelium
(Figure 4)
10 slides
4.1.3, Squamous cell carcinoma
(Figure 5) 10 slides
4.1.4, Pseudoepitheliomatous hyperplasia

\section{2, Selection Criteria:}

1. Only buccal mucosa was chosen ,to standardize the results in all the 4 study groups,as the fractal dimensions of different zones of oral mucosa vary considerably.

2. $\quad \mathrm{H} \& \mathrm{E}$ stained slides of all 4 study groups with confirmed diagnosis were included.

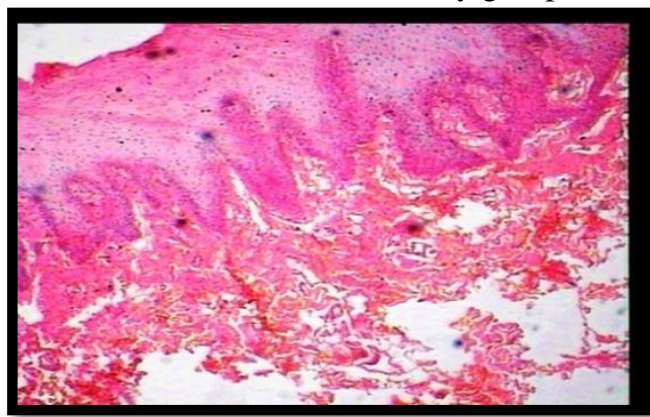

Figure 2 :Normal buccal mucosa(4X)

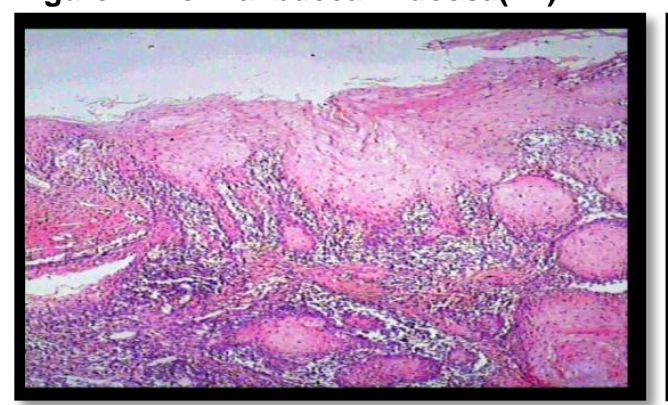

Figure 4 :Squamous cell carcinoma of buccal mucosa (4X)

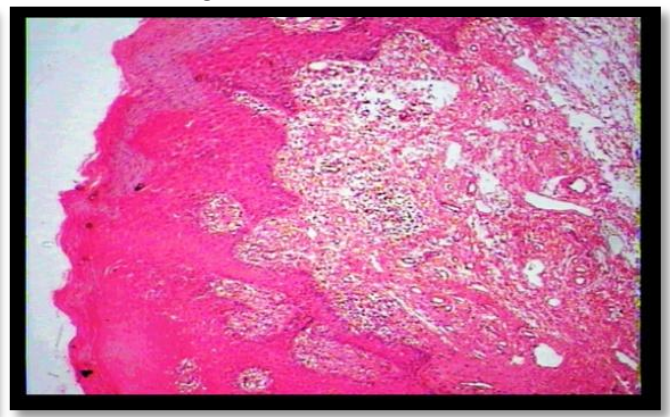

Figure 3:Dysplastic buccal mucosa(4X)

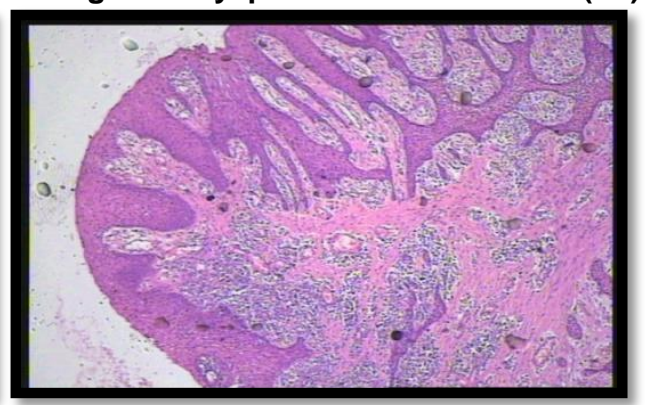

Figure 5:Pseudoepitheliomatous hyperplasia of buccal mucosa(4X) 


\section{3, Image J software with plugin for Frac Lac:}

To standardize our results and to avoid complications of multiple scans, we kept the box count from 2 to 12 only.Image analysis was performed to separate the epithelium from the connective tissue using an edge detecting filter software. Irregularity profile image was divided into boxes by the software \& output was regenerated in the graph form with the slope of the regression line generated from the software. The slope is the measure of fractal dimension.Figure 6.

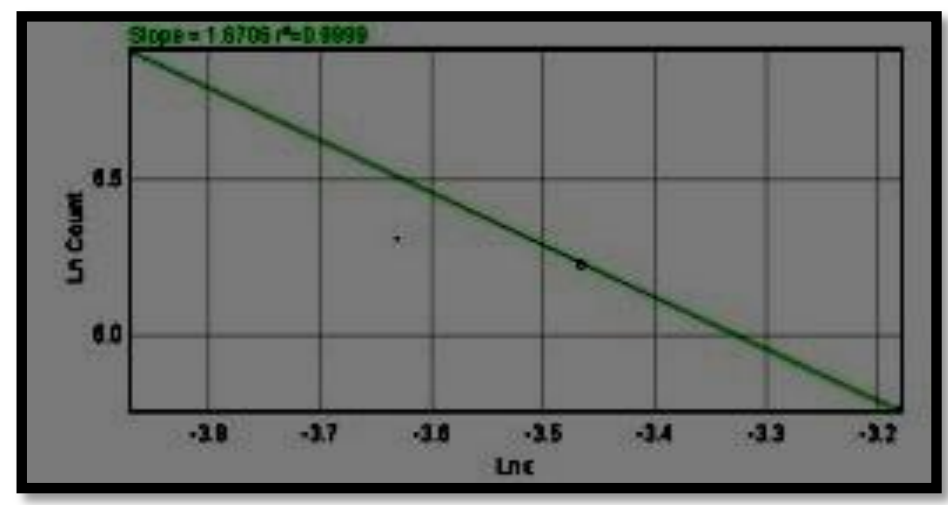

Figure 6:showing the slope $=$ fractal dimension $=1.6705$

The method was repeated 10 times at random places of a digital image throughout the epithelial connective tissue junction \& averaged to give the Fractal dimension of ECTI profile of a single image.The same method was repeated for all the images and the results were tabulated and compared.This study was completeted in 6 months duration.

\section{Results:}

\begin{tabular}{|l|l|l|l|l|}
\hline SR NO. & NORMAL & $\begin{array}{l}\text { PSEUDOEPITHELIOM-ATOUS } \\
\text { HYPERPLASIA } \\
\text { (PEH) }\end{array}$ & DYSPLASIA & OSCC \\
\hline 1. & 1.643 & 1.732 & 1.711 & 1.859 \\
\hline 2. & 1.523 & 1.643 & 1.773 & 1.888 \\
\hline 3. & 1.667 & 1.684 & 1.543 & 1.801 \\
\hline 4. & 1.587 & 1.514 & 1.699 & 1.758 \\
\hline 5. & 1.731 & 1.6432 & 1.743 & 1.841 \\
\hline 6. & 1.666 & 1.6788 & 1.766 & 1.785 \\
\hline 7. & 1.599 & 1.5551 & 1.769 & 1.777 \\
\hline 8. & 1.662 & 1.699 & 1.666 & 1.893 \\
\hline 9. & 1.691 & 1.721 & 1.722 & 1.871 \\
\hline 10. & 1.677 & 1.896 & 1.743 & 1.849 \\
\hline
\end{tabular}




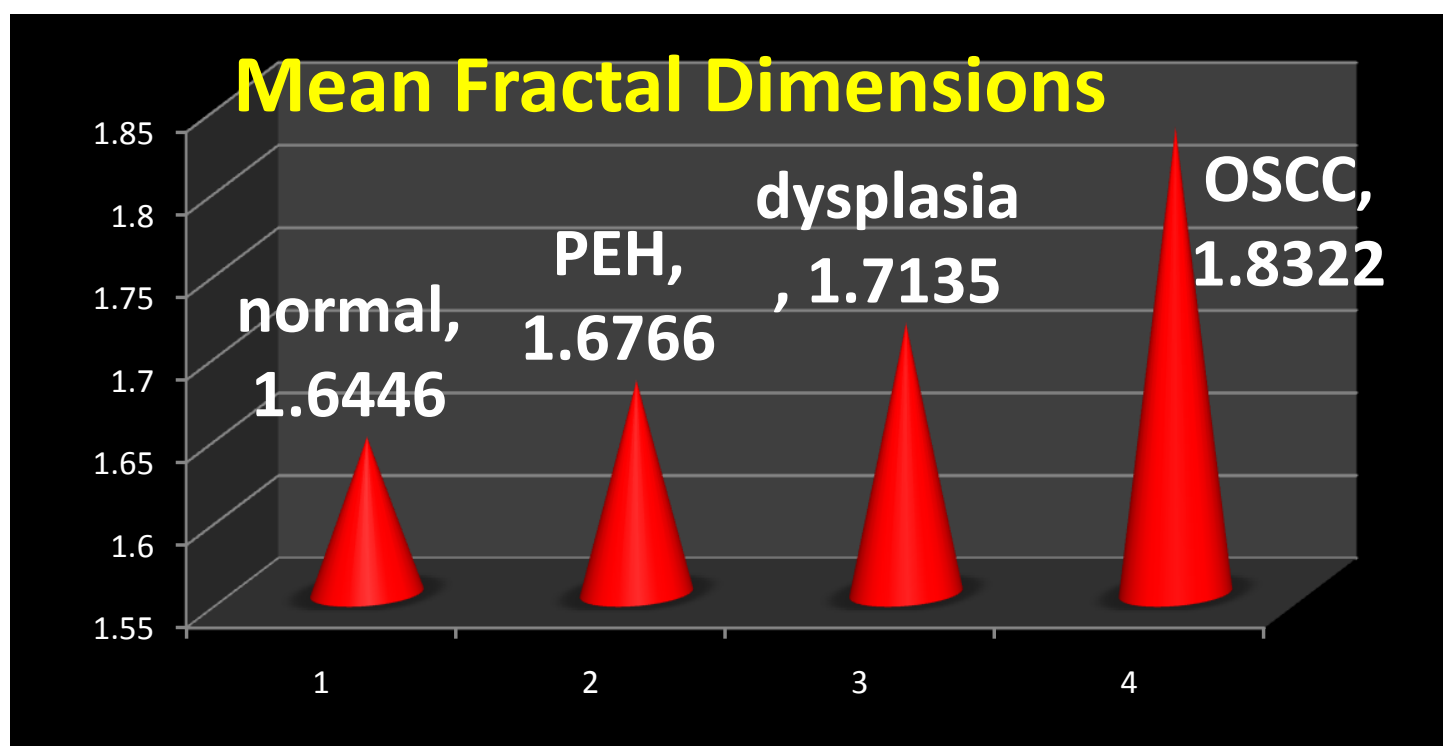

VI.

Discussion :

The increase in fractal dimensions is observed as the complexity increases from normal to pseudoepithelial hyperplasia then to dysplasia \& squamous cell carcinoma. The changes in the irregularity of ECTI profiles agree with the known mechanism of tumor growth.Defect in basement membrane \& loss of intercellular adhesion may be responsible \& contribute to the increased irregularity of tumor margins. Basement membranes ordinarily represent structural barriers to the passage of metabolic cells to and from the circulation. Further, since they surround epithelial, nerve and muscle tissue, they separate them from the surrounding stroma. Hence it acts as a barrier for the passage of malignant tumour cells that originate in these sites, as well as to cells invading these tissues that originated elsewhere The structural organisation of basement membrane is therefore useful in diagnostic histopathology to distinguish between dysplastic lesions, carcinoma in situ and invasive carcinoma ${ }^{6}$. Basement membranes appear to be crucial in tumour invasion and metastasis. Loss of basement membrane has been associated with many types of carcinomas, and with tumour cells in lymph node and organ metastases. While invasive tumours may retain their ability to synthesize basement membrane constituents, assembly is often defective, and other loss may be due to decreased synthesis or increased turnover of basement membrane components stimulated by tumour cell-derived proteases ${ }^{7}$. Cases with insitu carcinoma shows, microinvasion, thinning, fragmentation, and disruption of the basement membrane in the foci of microinvasion but not elsewhere. Many invasive tumours show variable retention of basal lamina proteins at their periphery. Furthermore, dysplastic and in situ lesions in a variety of sites may have discontinuous or interrupted basement membrane staining. Frequently, loss of basement membrane continuity cannot be used as a simple criterion of invasion ${ }^{8}$.

Collectively, these results highlight the relevance of shape-phenotype relationships that over three decades ago motivated Folkman and Moscona to ask, "how important shape is"? Ingber claimed that "the importance of cell shape appears to be that it represents a visual manifestation of an underlying balance of mechanical forces that in turn convey critical regulatory information to the cell". $9,10,11$ Thus, cell distortion influences cytoskeleton function and a cell's adhesion to the ECM. Cell-extracellular matrix (ECM) interactions are important for the survival and proliferation of normal epithelial cells. Squamous cell carcinoma (SCC) cells are known to be highly anchorage dependent indicating the importance of ECM interactions. In fact, SCC cells are characterized by their unrestrained growth, their ability to invade surrounding tissues, and their propensity to metastasize, thereby generating secondary tumors in organs distant from the primary tumor. Thus, the constituents of the ECM and their receptors, the integrins, must play prominent roles in both the regulation of normal epithelial growth and differentiation and the mechanisms of SCC tumor formation and metastasis. ECM molecules, as ligands for integrins, are essential in proper tissue development, adult tissue maintenance, wound healing, and oncogenesis. In the stratification of normal epithelium, proliferation is largely confined to the layer of cells attached to the basal lamina or basement membrane. This supportive matrix is composed of several components derived from keratinocytes and stromal cells or from the mutually cooperative interactions between these two cell populations. Altered expression of ECM molecules can contribute to oncogenetic development, hyperplastic growth, and tumor development. Therefore, changes in the integrin ligands appear to be essential for transformation of a pre-malignant squamous epithelium into a malignant lesion. The major ECM molecules implicated in SCC development include collagens, fibronectin, tenascin, and laminin. Thus cell shape and cytoskeletal structure appear to be tightly coupled to cell proliferation.In this way, tissue structure limits the 
constitutive ability of cells to proliferate. Within this framework it seems as if "Function Follows Form, And Not The Other Way Around"12,13.

\section{Limitations :}

The study is totally based on software \& digital photographic images.The ECTI profiles developed by the software is based on the colour pixel filter may give false positive $\&$ false negative results.The use of special stains like PAS or Masson's Trichrome stains to highlight the epithelial connective tissue junction requires further studies in this regards. This study is a short term study \& it should be evaluated on a large scale to check the accuracy of the results. The sample size in this study is too small to draw conclusions ,in this regards.

\section{Conclusion :}

It is clear that different measures of fractal geometry are useful in the study of both the physiological \& pathological changes in the oral mucosa. The tissue architectural changes manifested in the variations in the irregularity of the ECTI profiles can provide useful informations for understanding pathological changes.If fractal dimension range can be established in normal ,pseudoepithelial hyperplasia,dysplasia \& malignancy,a new grading system may emerge as an adjuvant aid in cancer diagnosis. The methodologies used in this study can be expanded to other pathological entities both inside \& outside the oral cavity combined with cellular morphometric analysis \& molecular analysis for further understanding of the pathological processes.

Dr. Gabriel Landini for guiding me throughout my study

Mr. Bhushan Mahajan to teach fraclac software.

Dentopath finder to provide slides of Peudoepitheliomatous Hyperplasia

\section{References :}

[1] Virchow, RLK. Cellular Pathology. London, UK: John Churchill; 1978. p. 204-7.1859. Special ed

[2] Rasha Abu Eid, Gabriel Landini, The Complexity of the Oral Mucosa: A Review of the Use of Fractal Geometry, CEAI, Vol.12, No.1, pp. 10-14, 2010

[3] Rasha Abu Eid, Gabriel Landini, Morphometrical differences between pseudo-epitheliomatous hyperplasia in granular cell tumours and squamous cell carcinomas, Histopathology, Volume 48 ,pages 407-416, March 2006

[4] Abu Eid, R. \& Landini, G. (2003) Quantification of the global and local complexity of the epithelial-connective tissue interface of normal, dysplastic, and neoplastic oral mucosae using digital imaging. Pathology Research and Practice, 475-82.

[5] Landini, G. (1996) Applications of fractal geometry in pathology. In: Iannaccone, P. \& Khokha, M. (Eds.) Fractal geometry in biological systems: An analytical approach. CRC Press, Boca Raton.

[6] Abrahamson Dale R. Recent studies on the structure and pathology of basement membranes. Journal of pathology 1986; 149: 257-278.

[7] Wilson F D, De-jun J, Pierce M A, Wiebkin W O. Oral cancer: role of the basement membrane in invasion. Australian dental journal 1999; 44; no 2; 93-97.

[8] Ardenne A J D. Use of basement membrane markers in tumour diagnosis. Journal of Clinical Pathology 1989; 42: 449-457.

[9] Folkman J, Moscona A. Role of cell shape in growth control. Nature. 1978; 273:345-349.

[10] Ingber DE. Mechanical control of tissue growth: function follows form. Proc Natl Acad Sci USA.2005; 102(33):11571-11572.

[11] Landini, G. \& Rippin, J. W. (1996) How important is tumour shape? Quantification of the epithelial-connective tissue interface in oral lesions using local connected fractal dimension analysis. Journal of Pathology, 179, $210-7$.

[12] Niimi, K., Yoshizawa, M., Nakajima, T. \& Saku, T. (2001) Vascular invasion in squamous cell carcinomas of human oral mucosa. Oral Oncology, 37, 357-64.

[13] Zioberl B L,Silverman S S, Kramer R H. Adhesive mechanisms regulating invasion and metastasis in oral cancer. Crit rev oral bio med. $2001 ; 12(6): 499-510$ 